\title{
Right-sided Diaphragm Rupture Incidentally Diagnosed by Coronal Views on Chest and Abdominal Computed Tomography
}

\author{
Seon Hee Kim, Chan Yong Park, Jung Joo Hwang, Hyun Min Cho \\ Department of Trauma Surgery, Pusan National University Hospital, Busan, Korea
}

A 57-year-old man was transferred to the emergency department of our trauma center because of decreased mentality. Chest computed tomography (CT) showed multiple rib fractures, aortic arch rupture, and suspicious diaphragm rupture. An intervention radiologist performed emergency thoracic endovascular aortic repair for traumatic aortic rupture. The coronal view of a follow-up abdominal CT scan revealed advanced herniation of the liver dome. The patient underwent a thoracoscopic examination and primary repair of the diaphragm injury through a thoracotomy under general anesthesia. A trauma team incidentally found traumatic diaphragm injury that would be easy to miss on routine examination and subsequently successfully managed the injury via surgical repair of the injured diaphragm without complication.

(Trauma Image Proced 2016(1):23-25)

Key Words: Diaphragm; Wounds and Injuries; Hernia; Multidetector Computed Tomography

\section{CASE}

A 57-year-old man was directly transported to our emergency department via the emergency medical service. The patient was found lying on the floor after a trauma. The initial chest computed tomography (CT) scan showed a suspicious diaphragm injury (Fig. 1.) with thoracic aortic injury. The trauma team called an intervention radiologist who performed emergency thoracic endovascular aortic repair for aortic rupture. A follow-up abdominal CT scan for liver injury was performed to obtain more detailed information regarding a suspicious diaphragm injury. The herniated liver dome was more prominent (Fig. 2.), and we decided on surgical repair of the injured diaphragm. The patient was sent to the operating room and underwent thoracoscopic examination under double-lumen general endotracheal anesthesia. We found traumatic diaphragm injury and herniation of the liver dome (Fig. 3.). The herniated liver dome was strangulated and had superficial laceration on the surface without active bleeding. We performed a lateral thoracotomy and repaired the injured diaphragm with nonabsorbable sutures in an airtight manner (Fig. 4.). The rightsided traumatic diaphragm injury was found incidentally through serial coronal views of chest and abdomen CT scans for evaluation of multiple severe injuries. The traumatic diaphragm injury was successfully managed through thoracoscopy and lateral thoracotomy under doublelumen general endotracheal anesthesia. The ruptured diaphragm could be tightly repaired without any

Received: April 8, 2016 Revised: June 11, 2016 Accepted: June 11, 2016

Correspondence to: Hyun Min Cho, Department of Trauma Surgery, Pusan National University Hospital, 179, Gudeok-ro, Seo-gu, Busan, Korea Tel: 82-51-240-7369, Fax: 82-51-240-7719, E-mail: csking1@daum.net

Copyright (c) 2016 Korean Association for Research, Procedures and Education on Trauma. All rights reserved.

@This is an open-access article distributed under the terms of the Creative Commons Attribution Non-Commercial License (http://creativecommons.org/ licenses/by-nc/4.0) which permits unrestricted noncommercial use, distribution, and reproduction in any medium, provided the original work is properly cited 


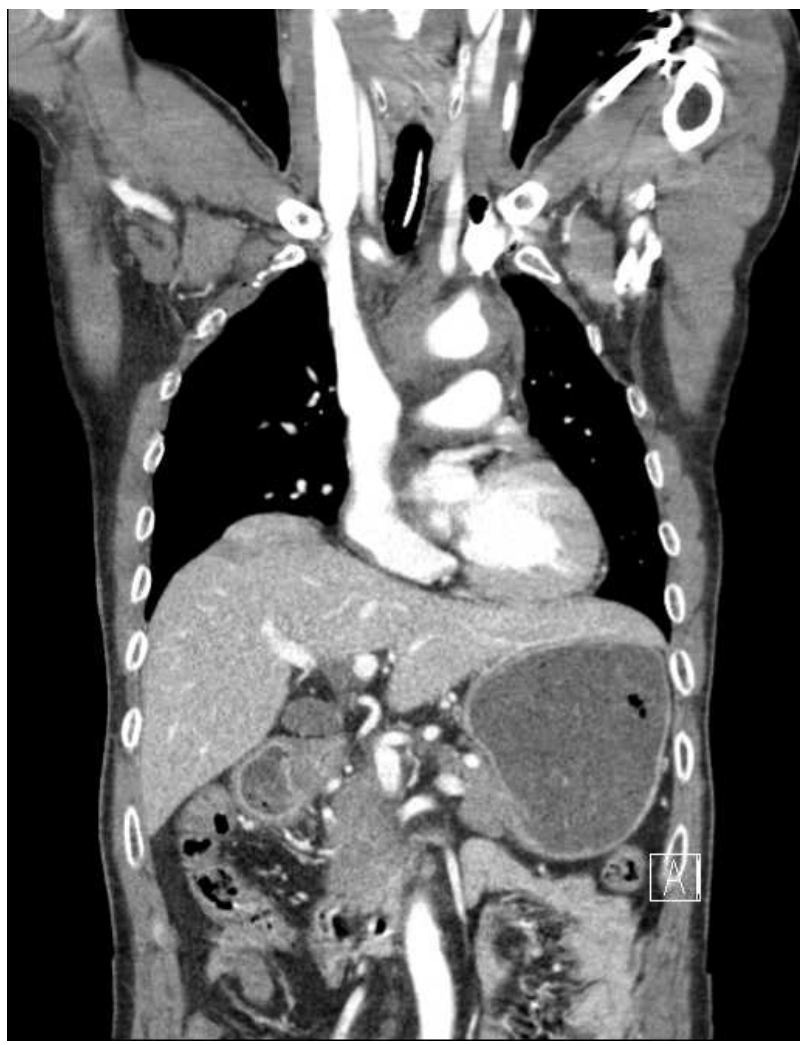

Fig. 1. The coronal view of the initial chest computed tomography scan shows a suspicious diaphragmatic injury at the medial portion of the right diaphragm.

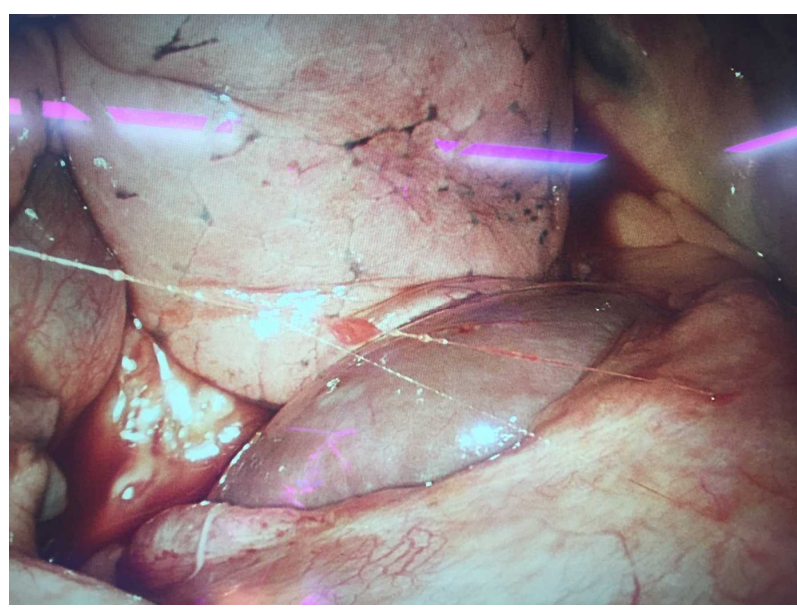

Fig. 3. Thoracoscopy under general anesthesia shows the strangulated liver dome due to the traumatic diaphragmatic rupture.

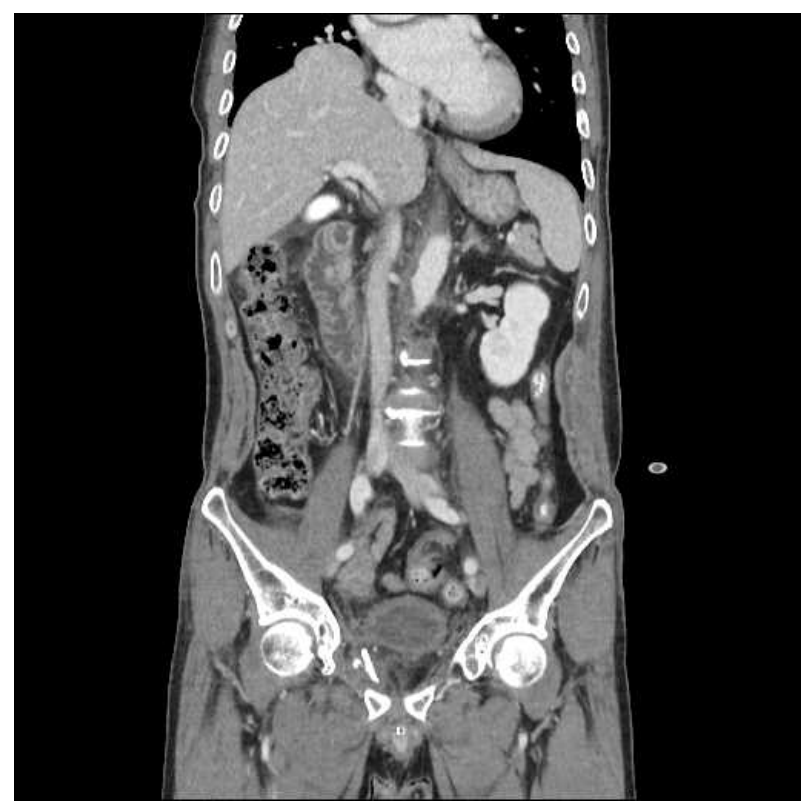

Fig. 2. The follow-up abdominal computed tomography scan reveals advanced herniation of the liver dome on a coronal view.

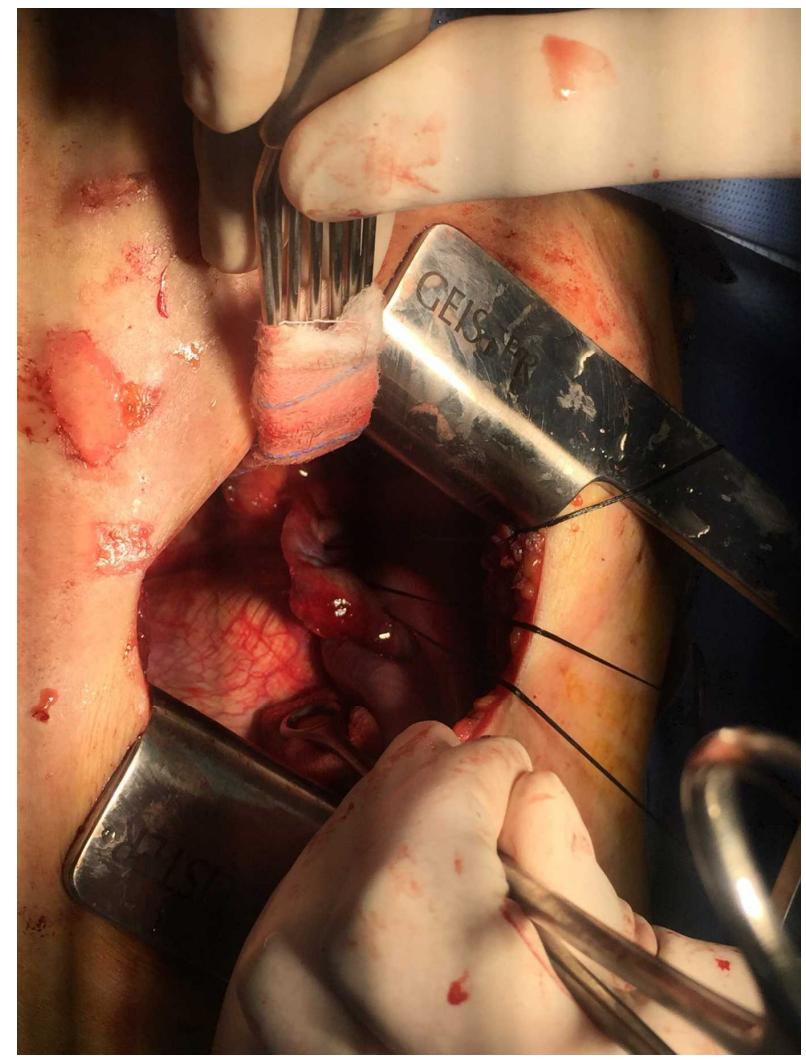

Fig. 4. The operative finding represents the restored liver dome into the abdomen and repaired diaphragm. 
complication owing to the early stage of injury.

\section{DISCUSSION}

In general, a right-sided diaphragm injury can be easily missed because the lesion is difficult to find and has no specific symptoms $(1,2)$. Because the specific diagnostic modality is not available in this situation, we have to adopt a conventional study for evaluation $(1,2)$. We found a traumatic diaphragm rupture incidentally through the comparison of serial coronal views on chest and abdomen CT scans. The traumatic diaphragm injury can be successfully managed through thoracoscopy and lateral thoracotomy under double-lumen general endotracheal anesthesia. It is important to suspect diaphragm injury, especially on the right side (2). The right-sided diaphragm injury is difficult with respect to diagnosis and management. The best way to solve the problem is initial suspicion and early detection of injury $(1,2)$.

\section{CONFLICT OF INTEREST}

No potential conflict of interest relevant to this article was reported.

\section{REFERENCES}

1. Kansal AP, Chopra V, Chahal AS, Grover CS, Singh H, Kansal S. Right-sided diaphragmatic eventration: A rare entity. Lung India : official organ of Indian Chest Society. 2009;26(2):48-50.

2. Panda A, Kumar A, Gamanagatti S, Patil A, Kumar S, Gupta A. Traumatic diaphragmatic injury: a review of CT signs and the difference between blunt and penetrating injury. Diagnostic and interventional radiology. 2014;20(2): 121-8. 